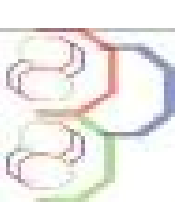

\title{
Succession des communautés végétales des jachères protégées en zone sahélienne: cas de Banizoumbou (Niger)
}

\author{
RABIOU Habou ${ }^{1 *}$, MAAZOU Rahila ${ }^{2}$, SOUMANA Idrissa1, MOUSSA MAMOUDOU Boubacar ${ }^{1}$, \\ ISSAHAROU-MATCHI Issiaka², MAHAMANE Ali \\ ${ }_{1}^{1}$ Faculté des Sciences Agronomiques, Université de Diffa, Niger \\ 2 Institut supérieur en Environnement et Écologie, Université de Diffa, Niger \\ ${ }^{3}$ Faculté des Sciences et Techniques, Université Abdou Moumouni de Niamey, Niger \\ Auteur correspondant : rabiouhabou@yahoo.fr
}

Original submitted in on $12^{\text {th }}$ December 2016. Published online at www.m.elewa.orgon $31^{\text {st }}$ March 2017 http://dx.doi.org/104314/jab.v111i1.11

\section{RÉSUMÉ}

Objectif : L'importance de la perturbation (en durée ou en intensité) semble décisive pour déterminer l'état de la végétation et la dynamique successionnelle. L'objectif de la présente étude est d'examiner les variations des paramètres écologiques des champs et des jachères protégées de Banizoumbou.

Méthodologie et résultats : Les relevés phyto-sociologiques ont été réalisés selon la méthode sigmatiste de Braun-Blanquet (1932). L'échantillonnage a été effectué sur la base de types des systèmes, ainsi les jachères et les champs ont fait l'objet d'inventaire suivant 6 transect, des placettes de $1000 \mathrm{~m}^{2}$ équidistantes de $500 \mathrm{~m}$ ont été placé. Ainsi au total 33 relevés ont été effectués dont 18 dans la jachère et 15 relevés dans le champ. II ressort de l'étude que la diversité dépend de stade successionnel même à l'intérieur d'un même type d'occupation. L'analyse des indices montre qu'en plus des jachères, les champs sont aussi d'importantes sources de biodiversité. Ainsi l'indice de diversité de Shannon évolue en fonction de l'âge de jachère et de champ, mais toute fois il évolue beaucoup plus rapidement au niveau de jachère que le champ. La densité moyenne de la zone d'étude est de 260 individus par hectare dont Guiera senegalensis représente plus de $70 \%$. La hauteur moyenne, le diamètre moyen, la densité moyenne et le recouvrement moyen les plus élevés sont observés au niveau des jachères.

Conclusion et application of résultats : la présente étude a montré que la variation de la diversité végétale est une fonction de l'âge aussi bien au niveau des champs que les jachères. Ainsi la diversité évolue plus rapidement au niveau des jachères.

Mots clés : Succession des communautés végétales, jachères protégées de Banizoumbou

\section{ABSTRACT}

Objective: The importance of disturbance (duration or intensity) seems decisive in determining the state of vegetation and successional dynamics. The purpose of this study is to study and examine variations in the floristic richness, diversity and production of protected fallow land in Banizoumbou.

Methodology and Results: The phyto-sociological surveys were carried out according to the sigmatist method of Braun-Blanquet (1932). Sampling was carried out on the basis of land use types, so fallows and fields were 

sahélienne : cas de Banizoumbou (Niger)

inventoried following 6 transect, 1000 square meter equidistant plots of $500 \mathrm{~m}$ were set. A total of 33 surveys were carried out, including 18 in the fallows and 15 in the fields. Our findings show that diversity depends on the successional stage even within the same type of land use. Analysis of the indices shows that in addition to fallow land, fields are also important sources of biodiversity. Thus, Shannon's diversity index increases according to the fallow and field age, but this increase is much higher in the fallows in than the fields. The results reveal also that average density of the study area is 260 individuals per hectare, of which Guiera senegalensis represents more than $70 \%$. The mean height, mean diameter, average density and highest average cover were observed at fallow land.

Conclusion and Application of results: The present study showed that variation in plant diversity is a function of age at both field and fallow land. Thus diversity evolves more rapidly at fallow land.

Key words: Succession of plant communities, protected fallow land of Banizoumbou

\section{INTRODUCTION}

La nature a mis des milliers d'années pour se doter d'une végétation climacique en harmonie avec son milieu, et l'homme lui, il n'a besoin que d'un très peu de temps pour l'effacer de la terre (Randrianjafy, 2009). La dégradation du paysage est, en général, caractérisée par l'appauvrissement des composants ligneux et l'enrichissement en éléments herbeux, c'est la dynamique régressive de la végétation. Les changements de modes d'utilisation du sol et leurs conséquences sur les systèmes écologiques ont été reconnus comme une composante majeure des changements globaux, d'une importance comparable aux changements climatiques et atmosphériques. Les sites de succession post-récolte sont quand même riches en espèces végétales notamment entre les herbacées et les arbres. C'est dans ces phases dynamiques qu'a lieu la recolonisation avec les espèces forestières et arbustes (Bogaert et Mahamane, 2005). Les écosystèmes protégés tels que les réserves et parcs sont identifiés pour prévenir la destruction ou dégradation des habitats et protéger les écosystèmes avec leurs populations riveraines. En Afrique tropicale, un système d'utilisation des terres très courant consiste en une phase de culture, suivie d'un abandon du champ dès qu'une baisse de rendement du travail se fait sentir. Cette phase de repos permet, en vingt à quarante ans, un retour à la savane originelle avec une bonne reconstitution des potentialités du sol et de la biodiversité ; en général, elle est mise à profit pour d'autres utilisations du milieu: pâturage de troupeaux domestiques, prélèvement de bois à usages divers (Barbier et al., 2006). Ce système, qui peut paraître archaïque, était en fait extrêmement bien adapté aux conditions de faible densité de population qui prévalaient dans ces régions jusqu'à une date récente (Serpantié et al., 2000). Actuellement, l'augmentation de la population, la tendance à la sédentarisation, la sécheresse, ont conduit à une forte augmentation des surfaces cultivées et à une diminution moyenne des temps de jachère, ce qui désorganise le système et bouleverse les paysages. Sous l'effet d'une mise en culture généralisée, les paysages Nigériens sont en évolution permanente. lls se présentent comme des mosaïques de champs cultivés, de jachères à divers stades de reconstitution et de zones incultivables ou protégées. La grande variété de physionomies et de compositions floristiques observée dans les jachères reflète les étapes du processus de succession postculturale et leurs diverses modalités en fonction des milieux et des régions (Achard et al., 2001). On sait que ces variabilités temporelles et spatiales sont sous le contrôle de divers facteurs, dont certains sont liés aux activités des hommes. Il est certain que le climat, les sols, les façons culturales, etc., ont un effet sur les successions, mais il est encore difficile de dire selon quelle hiérarchie s'organise leur influence. De même est-il difficile d'interpréter les variations de la richesse floristique, de la diversité et de la production dans ces milieux. La présente étude vise comme objectif global d'identifier les différentes communautés végétales en fonction de la durée de la jachère. De vérifier les processus par lequel les communautés végétales des jachères de Banizoumbou sont progressivement remplacée par d'autre en fonction de l'évolution de caractéristiques des jachères protégées. 


\section{MATÉRIEL ET MÉTHODES}

La zone d'étude : Le village de Banizoumbou est situé dans le degré carré de Niamey. Ce dernier se situe entre les 13 et 14 degrés de latitude Nord, et entre les 2 et 3 degrés de longitude Est II couvre une superficie de 11 $000 \mathrm{Km}^{2}$. Les caractéristiques générales du degré carré de Niamey sont typiques d'une grande bande zonale sahélienne (Traoré, 2007). Son relief est très peu marqué. II fait partie de l'actuel canton de Dantchiandou (Figure 1).

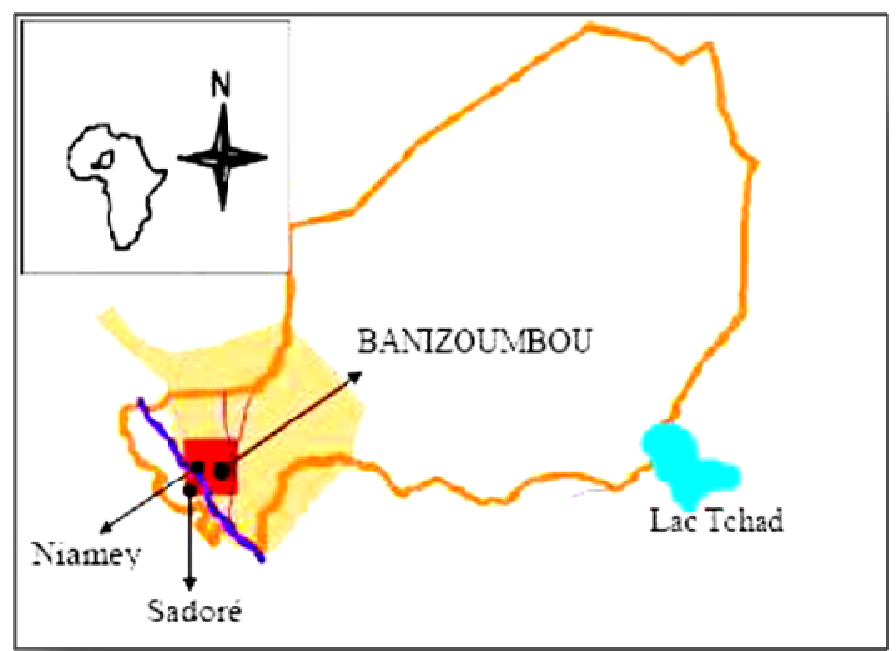

Figure 1 : Situation géographique de la zone d'étude (Banizoumbou) (Traoré, 2007).

La zone appartient au centre d'endémisme sahélien. Le climat de la zone est de type tropical sec. Les températures atteignent leur maximum en mars avril, juste avant les premières pluies de mai. Le village est situé entre les isohyètes $500-600 \mathrm{~mm}$. L'humidité relative de l'air connaît de fortes variations à l'échelle de l'année. La pluviométrie moyenne est $528,59 \pm 81,21$ pour les 20 dernières années. L'évapotranspiration potentielle atteint $2000 \mathrm{~mm}$ par an. Les vents sont de deux types dominants, l'Harmattan et la mousson. La sédimentation du bassin des lullemmeden est tabulaire. Dans sa partie ouest, ou se situe la zone d'étude, l'héritage d'une histoire climatique et géologique ancienne se traduit par la présence de nombreux plateaux latéritiques entrecoupes de vallées fossiles sableuses au fond desquelles serpentent des lits de rivières asséchés (Koris), côtoyant quelques dunes fixées, vestiges de l'alternance entre avancées du désert et périodes humides. L'absence de couches sédimentaires indurées et le faible dénivelé par rapport au niveau de base du fleuve n'a pas permis l'avènement de canyons spectaculaires. Bien au contraire, la monotonie du paysage est caractéristique, avec des dénivelés souvent très faibles entre les plateaux et les fonds de vallées ( $<60 \mathrm{~m}$, pentes moyennes de $1,3 \%$ ). La répartition des différentes formations végétales naturelles sur la zone d'étude tient à leur situation topographique et à leur affinité hydro-pédologique.
L'adaptation à l'insuffisance des réserves hydriques est à l'origine d'une colonisation par la "brousse tigrée" sur les entablements ferrugineux du Continental Terminal. Des faibles bandes de végétation denses collectent les écoulements générés par une large bande de sol nu en amont (Couteron et Lejeune, 2001). Dans la partie amont de la bande végétale qui recueille en priorité le ruissellement, se développent les plus grands individus de Combretum micranthum, Boscia angustifolia, Acacia macrostachya et Acacia ataxacantha (5 à $8 \mathrm{~m}$ selon Ehrmann, 1999). Les versants sableux sont colonisés par des steppes herbeuses ou arbustives à Combretacees. Au Combretum glutinosum s'associent diverses espèces plus sensibles aux nuances de l'édaphisme: Terminalia avicennoides pour les sols les plus secs, Combretum nigricans pour les sols les plus acides. Dans les aires cultivées et les jachères, Guiera senegalensis domine et cohabitant avec de plus rares Balanites aegyptiaca ou Faidherbia albida (Gao). Les graminées, Aristida mutabulis Cenchrus biflorus, et autres comme Zornia glochidiata, Mitracarpus scaber et Alysicarpus ovalifolius forment l'essentiel de la strate herbacée annuelle. Dans les bas-fonds argileux, lorsque l'engorgement n'est pas trop prononcé, la végétation des sols hydromorphes s'organise en fourrés denses : bois armes à Acacia nilotica, Acacia seyal, landes à buissons de Bauhinia rufescens et Piliostigma reticulatum. Selon le 
recensement de 2001, la population du village de Banizoumbou est de 1077 habitants dont 525 hommes et 552 femmes, regroupés au sein de 134 ménages, se partageant une superficie de $18 \mathrm{~km} 2$ avec un périmètre de $25 \mathrm{~km}$. II apparaît ainsi que la majeure partie des champs exploités par la population se trouve sur le terroir des villages limitrophes.

Collecte des données : Les relevés phytosociologiques ont été réalisés selon la méthode sigmatiste de BraunBlanquet (1932), l'échantillonnage est effectué sur la base de types des systèmes, ainsi les jachères et les champs ont fait l'objet d'inventaire. Les relevés ont été réalisés dans les champs et les jachères protégées de Banizoumbou. Suivant 6 transect, des placettes de 1000 $\mathrm{m}^{2}$ équidistantes de $500 \mathrm{~m}$ ont été placées. Au total 33 relevés ont été effectués dont 18 dans la jachère et 15 relevés dans le champ. Dans la jachère, 3 transects ont été placés avec un nombre variable des relevés, un transect dans la jachère de 1 an avec 5 relevés, un autre transect dans la jachère de 3 ans avec 5 relevés et un troisième transect dans une jachère de 5 ans avec 8 relevés. Dans le champ également 3 transects avec un nombre variable des relevés, un transect dans le champ de 1 an avec 5 relevés, un autre transect dans le champ de 3 ans avec 5 relevés et un troisième transect dans un champ de 5 ans avec 5 relevés. Pour chaque relevé, les variables stationnelles suivantes ont été notées : la croute structurelle, le type de systèmes, la litière, la présence de termitière. Dans chaque placette tous les individus ligneux ont fait l'objet des mesures afin d'analyser les paramètres dendrométriques. La hauteur, le diamètre des tiges et les diamètres perpendiculaires de chacun des individus ont été mesurés respectivement à l'aide de jalons gradués, d'un compas forestier et d'un mètre ruban.

Analyse et traitement des données: Les types biologiques ont été définis par Raunkiaer (1934) suivant la position des bourgeons et la taille des individus. L'analyse des formes d'adaptation des plantes permet une appréciation des conditions écologiques dans lesquelles elles vivent. Ces types biologiques par leur répartition, traduisent fidèlement les conditions écologiques d'une région et leur étude permet d'avoir une idée de la végétation d'une région donnée (Mahamane, 2005). Dans le cadre de ce travail, les catégories ont été définies suivant la classification de Raunkiaer (1934). Les types phytogéographiques adoptés sont des subdivisions chorologiques généralement admises pour l'Afrique (White, 1986) et déjà largement utilisés (Mahamane, 2005). II s'agit de : Espèces à distribution limitée au continent africain Soudano-Zambéziennes (SZ) : espèces distribuées à la fois dans les centres régionaux d'endémisme soudanien et zambézien ; GuinéoCongolaises (GC) : espèces distribuées dans la région guinéenne ; Sah.S: Saharo-sahélien; Med: méditerranéen. La diversité spécifique rend compte de la richesse et de la distribution d'abondance spécifique des phytocénoses. Elle est définie par la richesse spécifique et l'indice de diversité de Shannon. La richesse spécifique d'une communauté est le nombre d'espèces que contient cette communauté. Quant à l'indice de Shannon et Weaner, il est défini par :

$$
H=-\sum_{i=1}^{s} p_{i} \log _{2} p_{i} \quad \mathrm{Eq}=\frac{\mathrm{H}}{\mathrm{Hmax}} \text { avec } \operatorname{Hmax}=\log _{2} \mathrm{~S}
$$

Avec : $H$ = indice de diversité spécifique de Shannon et Weaner $; p i=$ ni/ $N$ avec ni fréquence d'individu d'une espèce i et $\mathrm{N}$ la somme des fréquences de toutes les espèces.

$\mathrm{Eq}$ : Equitabilité ou régularité, $S$ désigne la richesse spécifique et $\log _{2} S$ la diversité maximale.

La distribution de Weibull à 3 paramètres $(a, b$ et $c)$ se caractérise par une grande souplesse d'emploi et une grande variabilité de forme. Sa fonction de densité de

$$
f(x)=\frac{c}{b\left[\frac{x-a}{b}\right]}^{a-1} e^{-\left[\frac{[x-a g}{b}\right]^{2}}
$$

probabilité, $f(x)$ se présente sous la forme ci-dessous (Rondeux, 1999). 


\section{RÉSULTATS}

Caractéristiques globales de la flore : Au total de 33 relevés ont été effectué avec un total de 50 espèces dont 40 espèces herbacées et 10 espèces ligneuses réparties dans39 genres et regroupées dans 17 familles. L'analyse de la diversité alpha montre que la zone d'étude est relativement diversifiée avec un indice de Shannon $\mathrm{H}$ égale à 4,48 et une régularité $E$ égale à 0,8 . Les familles les mieux représentées sont les Poaceae avec 15 espèces soit $31 \%$ suivies de Leguminoseaepapillonaceae avec 6 espèces soit 12\%. Les Convolvulaceae, les Combretaceae et les Cyperaceae sont respectivement représentées par 4, 4, 3 espèces soit $8 \%, 8 \%$ et $6 \%$. Les familles les moins représentées ont entre 1 et 2 espèces et représentent au total $35 \%$ (Figure 2).

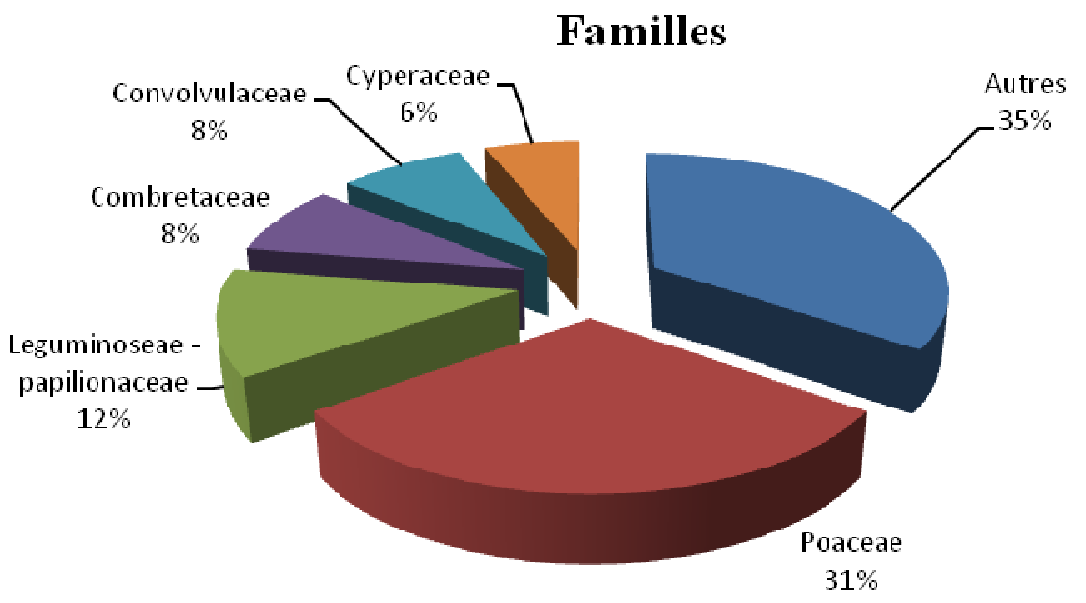

Figure 2 : Composition floristique

Analyse globale des types biologiques : L'analyse du graphiques des types biologiques montre que les thérophytes sont les formes les mieux représentées avec $65,3 \%$, suivis des phanérophytes avec $22,44 \%$. Par contre les chaméphytes et les géophytes sont très peu représentés avec respectivement 4,08\% et 2,04\% (Figure 3). La prédominance des thérophytes et les phanérophytes témoin de l'appartenance de la zone d'étude à une zone aride.

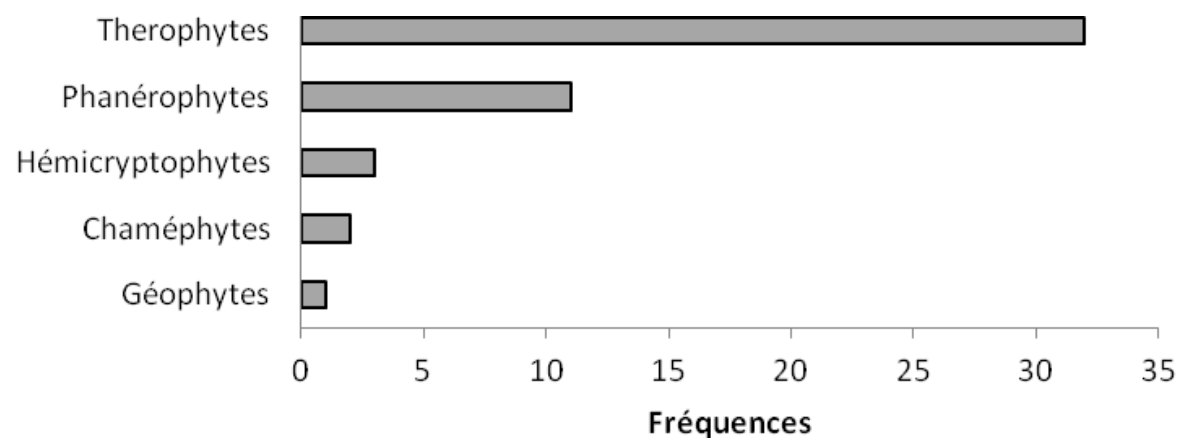

Figure 3. Spectre de types biologiques 
Analyse globale des types phytogéographiques: L'analyse du graphique résumant la distribution chorologique des différentes espèces montre une prédominance des espèces Guinéo-congolaise-soudano- zambéziennes avec $35,41 \%$ suivies des soudanozambéziennes avec $29,1 \%$. Par contre les espèces Saharo-sindiennes-méditerranéens sont très peu représentées (Figure 4).

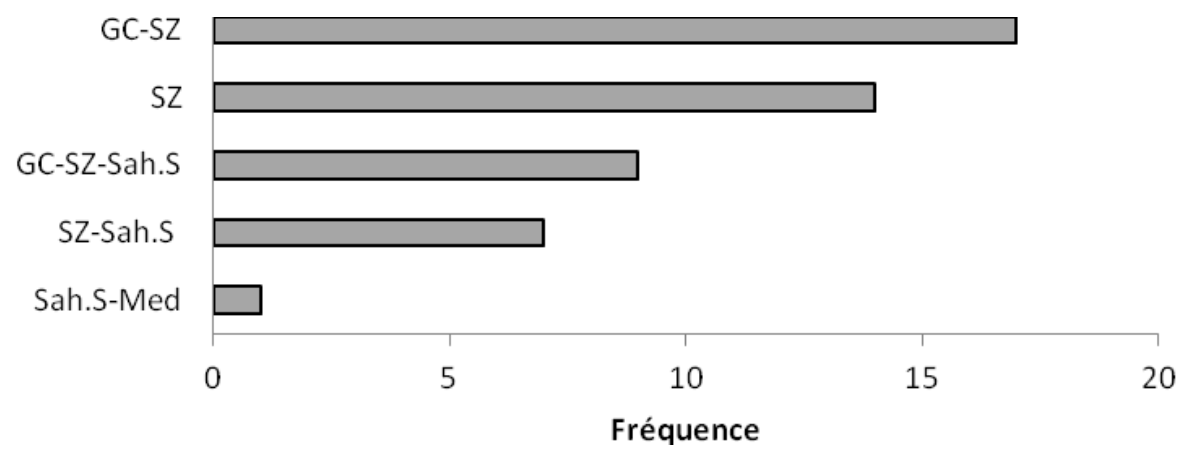

Figure 4 : Spectre de Types phytogéographiques

Analyses des types biologiques, phytogéographiques et diversité des jachères

Analyse des types biologiques des jachères : Afin de décerner la dynamique des communautés végétales des jachères il est important de connaitre les processus qui gouvernent cette dynamique depuis bien avant l'abandon des champs donc leurs mise en jachère. II ressort donc de l'analyse du tableau que les Thérophytes sont largement les plus représentées suivies des Phanérophytes quelques soit l'âge de la jachère (Tableau 1). Cependant les Chaméphytes, et les géophytes ne sont observées que dans la jachère de 1 an et de 5 ans avec des très faibles proportions $2,7 \%$ chacune. Dans la jachère de 5 ans nous avons observé la présence de plus grand nombre de types biologiques ce qui explique une évolution progressive de la succession post culturale.

Tableau 1. Types biologiques des jachères

\begin{tabular}{l|c|c|c|c|c|c}
\hline \multirow{2}{*}{} & \multicolumn{2}{|c|}{ Jachère_1 an } & \multicolumn{2}{c|}{ Jachère_3 ans } & \multicolumn{2}{c}{ Jachère_5 ans } \\
\cline { 2 - 7 } & Nombre & $\%$ & Nombre & $\%$ & Nombre & $\%$ \\
\hline Chaméphytes & 1 & 3,4 & 0 & 0 & 1 & 2,7 \\
Géophytes & 1 & 3,4 & 0 & 0 & 1 & 2,7 \\
Hémicryptophyte & 0 & 0 & 0 & 0 & 1 & 2,7 \\
Phanérophytes & 6 & 20,6 & 4 & 13,7 & 9 & 25 \\
Thérophytes & 21 & 72,4 & 25 & 86,2 & 24 & 66,6 \\
\hline \hline Total & 29 & 100 & 29 & 100 & 36 & 100 \\
\hline
\end{tabular}

Analyse des types phytogéographiques des jachères: L'analyse du tableau 6 de l'affinité chorologique des espèces de la jachère fait ressortir la dominance des espèces guinéo-congolaises-soudanozambéziennes quelques soit l'âge de la jachère (Tableau
2). Cependant les proportions des soudanozambéziennes et guinéo-congolaise- soudanozambézienne-saharo-sindiennes sont indépendantes de l'âge de jachère. 
Rabiou et al., J. Appl. Biosci. 2017 Succession des communautés végétales des jachères protégées en zone sahélienne : cas de Banizoumbou (Niger)

Tableau 2. Types phytogéographiques des jachères

\begin{tabular}{l|c|c|c|c|c|c}
\hline \multirow{2}{*}{} & \multicolumn{2}{|c|}{ Jachère_1 an } & \multicolumn{2}{c|}{ Jachère_3 ans } & \multicolumn{2}{c}{ Jachère_5 ans } \\
\cline { 2 - 7 } & Nombre & $\%$ & Nombre & $\%$ & Nombre & $\%$ \\
\hline SZ & 8 & 28,57 & 7 & 24,13 & 10 & 28,57 \\
GC-SZ & 11 & 39,28 & 14 & 48,27 & 16 & 45,71 \\
GC-SZ-Sah.S & 6 & 21,42 & 5 & 17,24 & 6 & 17,14 \\
SZ-Sah.S & 3 & 10,71 & 3 & 10,34 & 3 & 8,57 \\
\hline \hline Total & 28 & 100 & 29 & 100 & 35 & 100 \\
\hline
\end{tabular}

Indices de diversités de jachère: Lorsqu'on tient compte du seuil de variation de l'indice de ShannonWeaver ( 0 à 5$)$, on peut dire que tous les différents stades de jachères sont relativement diversifiés car les valeurs calculées sont supérieures à 3,4 bits (Tableau 3).
Cependant, l'indice augmente avec l'âge de la jachère et la régularité reste constante. La diversité spécifique est relativement plus importante dans la jachère de 5 ans que dans la jachère de 1 an et de 3 ans.

Tableau 3. Indices de diversités des jachères

\begin{tabular}{l|c|c|c}
\hline \multicolumn{1}{c|}{ Indice } & Jachère $\mathbf{1}$ an & Jachère $\mathbf{3}$ ans & Jachère $\mathbf{5}$ ans \\
\hline $\mathrm{H}$ & 3,96 & 4,19 & 4,27 \\
$\mathrm{H} \max$ & 4,85 & 4,85 & 5,20 \\
$\mathrm{~S}$ & 29 & 29 & 37 \\
$\mathrm{R}$ & 0,81 & 0,86 & 0,82 \\
\hline
\end{tabular}

Analyses des types biologiques, phytogéographiques et diversité des champs Analyse des types biologiques des champs: Les champs sont des excellents refuges de la biodiversité. En effet, la fumure qu'on apporte au champ améliore la fertilité, le sarclage ameubli le sol et crée des conditions écologiques favorable à l'installation des diverses espèces. II ressort donc de l'analyse du tableau que les
Thérophytes sont largement les plus représentées suivi des Phanérophytes quelques soit l'âge de champ comme dans le cas de jachère (Tableau 4). Cependant les Chaméphytes et les géophytes ne sont observées que dans le champ de 5 ans. D'une manière générale les champs de 5 ans présentent la plus grande diversité de types biologiques.

Tableau 4 : Types biologiques des champs

\begin{tabular}{l|c|c|c|c|c|c}
\hline \multirow{2}{*}{} & \multicolumn{2}{|c|}{ champ 1 an } & \multicolumn{2}{c|}{ champ 3 ans } & \multicolumn{2}{c}{ champ 5 ans } \\
\cline { 2 - 7 } & Nombre & $\%$ & Nombre & $\%$ & Nombre & $\%$ \\
\hline Chaméphytes & 0 & 0 & 0 & 0 & 1 & 3,57 \\
Géophytes & 0 & 0 & 0 & 0 & 2 & 7,14 \\
Hémicryptophytes & 0 & 0 & 2 & 6,66 & 1 & 3,57 \\
Phanérophytes & 4 & 18,18 & 6 & 20 & 5 & 17,85 \\
Thérophytes & 18 & 81,81 & 22 & 73,3 & 19 & 67,85 \\
\hline \hline Total & 22 & 100 & 30 & 100 & 28 & 100 \\
\hline
\end{tabular}

Analyse des types biogéographiques des champs: L'analyse du tableau de types phytogéographiques des espèces de champ montre la dominance des espèces guinéo-congolaises-soudano-zambéziennes quelques soit l'âge de champ (Tableau 5). Cependant les proportions des soudano-zambéziennes augmentent avec l'âge de champ. Par contre les espèces soudanozambéziennes sont indépendantes de l'âge de champ. 
Rabiou et al., J. Appl. Biosci. 2017 Succession des communautés végétales des jachères protégées en zone sahélienne : cas de Banizoumbou (Niger)

Tableau 5. Types phytogéographiques des champs

\begin{tabular}{l|c|c|c|c|c|c}
\hline & \multicolumn{2}{|c|}{ Champ 1 an } & \multicolumn{2}{c|}{ Champ 3 ans } & \multicolumn{2}{c}{ Champ 5 ans } \\
\cline { 2 - 7 } & Nombre & $\%$ & Nombre & $\%$ & Nombre & $\%$ \\
\hline SZ & 4 & 19,04 & 7 & 24,13 & 8 & 32 \\
GC-SZ & 8 & 38,09 & 15 & 51,72 & 11 & 44 \\
GC-SZ-Sah.S & 6 & 28,57 & 4 & 13,79 & 4 & 16 \\
SZ-Sah.S & 3 & 14,28 & 3 & 10,34 & 2 & 8 \\
\hline \hline Total & 21 & 100 & 29 & 100 & 25 & 100 \\
\hline
\end{tabular}

Indices de diversités de champ: La variation de l'indice de Shannon-Weaver, montre que tous les différents stades de champ présentent des diversités relativement bonnes car les valeurs calculées de l'indice sont supérieures à 3,4 bits (Tableau 6). Cependant, l'indice augmente avec l'âge de champ et la régularité reste autour de 0,8 quelques soit l'âge de champ. La richesse spécifique est relativement plus importante dans le champ de 3 ans.

Tableau 6. Indices de diversités des champs

\begin{tabular}{l|l|l|l}
\hline Indices & Champ 1 an & Champ 3 ans & Champ 5 ans \\
\hline $\mathrm{H}$ & 3,96 & 3,97 & 3,98 \\
$\mathrm{H} \max$ & 4,45 & 4,95 & 4,7 \\
$\mathrm{~S}$ & 22 & 31 & 26 \\
$\mathrm{R}$ & 0,8 & 0,8 & 0,84 \\
\hline
\end{tabular}

Caractéristiques dendrométriques: La densité moyenne (nombre d'individus par hectare) de la zone d'étude est de 260 individus par hectare dont Guiera senegalensis représente plus de $70 \%$. Le recouvrement moyen de la zone d'étude est de 6,08\%. La hauteur moyenne de la végétation ligneuse est de 1,28 $\pm 0,64 \mathrm{~m}$. La hauteur moyenne la plus élevée est observée dans la jachère est de 1,39 $\pm 0,63$ alors qu'elle est de 1,01 $\pm 0,5$ dans le champ. Le diamètre moyen le plus élevé s'observe également dans la jachère $4,48 \pm 1,9$. Les valeurs élevées de densité (nombre d'individus par hectare), de recouvrement moyen, de diamètre moyen de houppier sont observés dans la jachère avec respectivement 194, 3,06\%, 1,20 00,74 (Tableau 7). Cela témoin la mise en place de mécanisme de succession post-cultural.

Tableau 7 : Caractéristiques dendrométriques

\begin{tabular}{l|l|l|l|l|l}
\hline & $\begin{array}{l}\text { Recouvrement } \\
\text { moyen }(\%)\end{array}$ & $\begin{array}{l}\text { Diamètre moyen du } \\
\text { Houppier }(\mathbf{m})\end{array}$ & $\begin{array}{l}\text { Densité } \\
\text { (individus/ha) }\end{array}$ & $\begin{array}{l}\text { hauteur } \\
\text { moyenne }(\mathbf{m})\end{array}$ & $\begin{array}{l}\text { diamètre } \\
\text { moyen }(\mathbf{c m})\end{array}$ \\
\hline champ & $3,06 \mathrm{a}$ & $1,20 \pm 0,74 \mathrm{a}$ & $194 \mathrm{a}$ & $1,01 \pm 0,5 \mathrm{a}$ & $3,07 \pm 1,83 \mathrm{a}$ \\
jachère & $9,11 \mathrm{~b}$ & $1,65 \pm 0,9 \mathrm{~b}$ & $326,11 \mathrm{~b}$ & $1,39 \pm 0,63 \mathrm{~b}$ & $4,48 \pm 1,9 \mathrm{~b}$ \\
\hline Probabilité & 0,001 & 0,006 & 0,001 & 0,03 & 0,04 \\
\hline
\end{tabular}

Analyse comparée de la structure en diamètre : Les structures en diamètre de la jachère et de champ présentent des distributions plus ou moins différentes. En effet, dans la jachère la distribution est en « J renversée "caractéristique des peuplements multispécifiques ou inéquiennes avec un paramètre $c$ de la distribution théorique de Weibull égale à 0,79 . Par contre dans le champ, la structure en diamètre présente une distribution asymétrique négative, caractéristiques des peuplements mono-spécifiques avec une prédominance d'individus jeunes ou de petits diamètres, avec un paramètre de forme $c$ de la distribution théorique de Weibull égale à 1,71 proche de celui de tout le peuplement (Figure 5). 


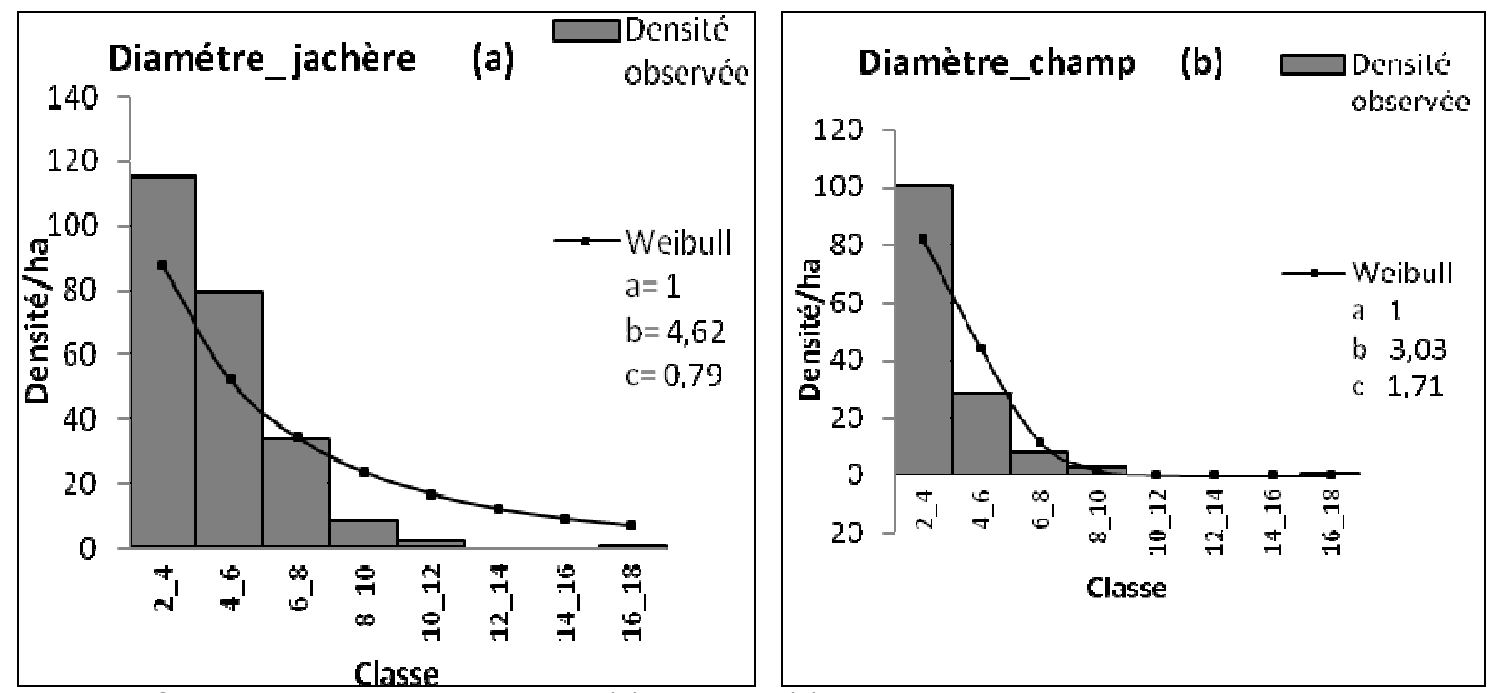

Figure 5. Structure en diamètre de jachère $(a)$ et champ (b)

Analyse comparée de la structure en hauteur: Les structures en hauteur de la jachère et de champ présentent tous les deux des distributions asymétriques négative, caractéristique des peuplements mono- spécifiques avec une prédominance d'individus jeunes ou de petits diamètres, avec un paramètre de forme $c$ de la distribution théorique de Weibull égale à 2,31 dans la jachère et 1,88 dans le champ (Figure 6).

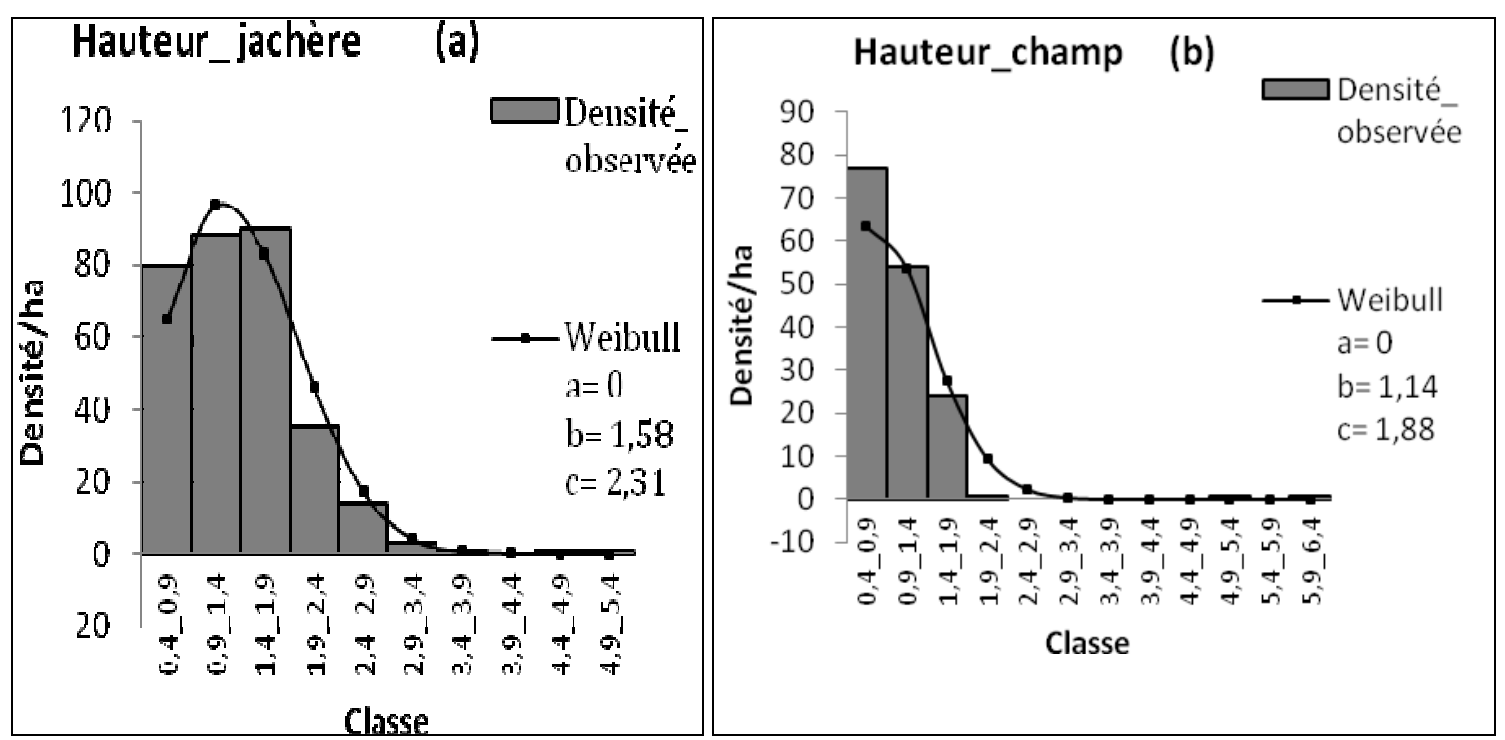

Figure 6 : Structure en hauteur de jachère (a) et champ (b)

\section{DISCUSSION}

L'agriculture traditionnelle se conforme généralement à un système comprenant une phase de culture suivie d'une phase de repos. Après l'abandon du champ, la végétation se reconstitue par un processus de succession secondaire. Un tel système d'exploitation induit un paysage en mosaïque ; celle-ci, en perpétuelle évolution, est plus ou moins complexe et morcelée selon la taille et l'agencement des parcelles et des zones non cultivées. Selon Tijani (2008) dans le Fakara, les jachères occupent relativement une faible proportion de l'espace (23\% contre $58 \%$ pour les champs cultivés) et présentent un couvert végétal (herbacé et arbustif) relativement dense. Ainsi notre zone d'étude est fortement fragilisée par des pressions anthropiques dans un contexte de péjoration 

sahélienne : cas de Banizoumbou (Niger)

climatique, ce qui se traduit par des conséquences sur la dynamique des communautés végétales. L'analyse floristique a permis de recenser 17 familles en 39 genres et 50 espèces ( 40 herbacées et 10 ligneuses). Une telle flore est caractéristique des zones purement arides. Une forte thérophytisation des espèces est observée à hauteur de $65,3 \%$ de la flore du terroir. Cette tendance est donc la signature d'une stratégie d'adaptation aux précarités climatiques dans la zone d'étude. L'étude chorologique montre que ce sont les espèces Guinéocongolaises-soudano-zambéziennes qui dominent avec $35,41 \%$, suivies des soudano-zambéziennes avec $29,1 \%$. Ces résultats sont similaires à ceux obtenus par Mariama (2009) dans la même zone. Selon qui les espèces Guinéo-congolaises-soudano-zambéziennes dominent à $39,58 \%$, suivies des soudano-zambéziennes avec 26,71 $\%$. Le coefficient générique a une valeur de 0,78 . Les indices globaux de diversité de Shannon d'une part et d'équitabilité de Pielou d'autre part calculés sont respectivement de 4,48 et 0,8 bits. Ces valeurs des indices de diversités montrent que la flore de la zone d'étude est riche. Mais elles dépendent de type d'occupation de sol selon qu'on soit dans la jachère et dans le champ. La diversité dépend aussi de stades successionnels même à l'intérieur d'un même type d'occupation. L'analyse de ces indices montre aussi que les champs sont aussi dimportantes sources de biodiversité (3,9 bits). Ainsi, l'indice de diversité de Shannon évolue en fonction de l'âge de jachère et de champ, mais toute fois, il évolue beaucoup plus rapidement au niveau de jachère que le champ (Tableau 3 et 6). Dans certains travaux, la distinction a été introduite entre "champs de case» et "champs de brousse». Elle apparaît comme très explicative de la variabilité de la végétation dans les jachères de moins de six ans de la région de Bondoukuy, au Burkina Faso (Hien, 1996) ainsi que dans les milieux post-culturaux du Mali (Yossi, 1996). Les champs de case, très proches des villages, sont cultivés plus longuement (généralement plus de dix ans) que les champs de brousse ; les jachères qui en sont issues sont soumises à des pressions anthropiques bien plus fortes (nombreuses déjections qui modifient le milieu, piétinement, pâturage intense, etc.). Une flore bien différenciée s'associe à chaque type de milieu, par exemple les ligneux Dichrostachys cinerea, Ziziphus mauritiana et Combretum fragrans sont caractéristiques des champs de case dans le terroir de Missira, au Mali. La présente étude confirme la dynamique des communautés végétales dans les successions post-culturales dans la Commune de Banizoumbou. Ainsi les caractéristiques écologiques déterminées dans cette étude représentent des marqueurs de types d'occupations des terres. Bien que la mise en culture perturbe fortement la végétation, la variabilité des pratiques culturales n'est pas déterminante dans la variabilité floristique des jachères (Fournier et al., 2001). L'utilisation du milieu par l'homme (modes culturaux au sens large, pâturage, cueillette) conditionne cependant très rapidement la reconstitution de la jachère et le développement de certains types de végétation notamment à dominance des herbacées pérennes (Song et al., 2011). Plus l'utilisation agricole a modifié le sol par rapport à celui de milieu naturel plus donc la reconstitution est difficile; ainsi une longue durée de culture et un labour à la charrue ont une influence négative. Dans l'optique de mettre en évidence la succession des communautés végétales dans les jachères, il a été nécessaire de mettre en évidence les communautés végétales des champs afin de faire une comparaison. Ainsi les indices des diversités les plus importants sont observés au niveau des jachères, et augmente en fonction de l'âge de jachère. Ces résultats suggèrent que la jachère est plus diversifiée que le champ. La colonisation par des nouvelles espèces augmente la diversité et elle-même fonction de l'âge. Des études de la végétation réalisées dans la zone soudanienne par Fournier et al. (2001) sur les jachères ont montré que pendant les premières années de jachère diverses herbes dominent le milieu sous l'effet conjoint des facteurs pédologiques et anthropiques. Mais Camara (1997) a montré qu'après dix ans d'abandon environ, la graminée pérenne Andropogon gayanus remplace ces espèces et devient dominante dans les jachères, quel que soit le sol. Dans le cas de notre travail, nous n'avons noté la présence de l'espèce Andropogon gayanus. L'explication réside dans deux faits majeurs. En effet, la différence des conditions agro-écologiques entre la zone soudanienne et zone sahélienne (notre zone d'étude), notamment la pluviométrie d'une part et d'autre part l'âge nécessaire pour l'apparition de Andropogon gayanus. Dans le cas de la présente étude il a été effectué des relevés dans les jachères de 1 an de 3 ans à 5 ans, alors que Andropogon gayanus commence son apparition après dix ans. La composition floristique est un excellent indicateur des conditions édaphiques, le lien réciproque entre végétation et sol est connu depuis longtemps en écologie végétale et plus spécialement en phytosociologie). Ce lien est très visible dans la zone de Banizoumbou. En effet, le groupement à Guiera senegalensis et Mitracarpus scaber domine les champs, néanmoins ces espèce sont beaucoup présentent dans la jachère aussi. Dans la plus part des cas, c'est la simple 

sahélienne : cas de Banizoumbou (Niger)

présence de ces espèces qui a un caractère indicateur. Mais parfois les espèces indicatrices sont en même temps des espèces dominantes qui constituent le groupement. La zone de Banizoumbou est une zone de transition régionale du Sahel, elle présente une zone de plateau à brousse, ces zone se raccordent au long glacis caractérisé par des champs et des jachères. De manière générale la végétation est proche de la formation à Combretum glutinosum et Guiera senegalensis sur sols gréseux décrite par White (1986) pour le Jebel Marra dans la zone de transition régionale du Sahel. Dans tous les milieux post-culturaux de la région, l'arbuste Guiera senegalensis domine strictement la strate ligneuse, d'ailleurs extrêmement pauvre. C'est seulement dans les stations les plus humides qu'ils sont remplacés par Piliostigma reticulatum, Combretum glutinosum, Annona senegalensis ou Hyphaene thebaica (Delabre, 1998). Durant les cinq premières années d'abandon cultural, les ligneux qui étaient présents sous forme de rejets des souches lors de la mise en jachère augmentent en taille, mais il n'y a que peu de recrutement. Au-delà de cinq ans, taille et phyto volume diminuent, témoignant du vieillissement de la population. La flore herbacée, pauvre elle aussi, est dominée par un groupe d'espèces ubiquistes (Zornia glochidiata, Eragrostis tremula, Mitracarpus scaber) pendant toute la phase postculturale. Durant les cinq premières années d'abandon cultural, les ligneux qui étaient présents sous forme de rejets des souches lors de la mise en jachère augmentent en taille, mais il n'y a que peu de recrutement. Les graminées pérennes, représentées presque exclusivement par Andropogon gayanus, restent très peu abondantes, même aux stades âgés. En fait, la plupart des vieilles jachères correspondent à un état dégradé par l'homme, où la dynamique de succession ne s'exprime qu'imparfaitement. Des observations analogues ont été faites sous un climat semblable à Dalombedougou au Mali (Cissé et al., 1993) et dans le delta intérieur du Niger (Hiernaux, 1983). La dynamique progressive de la végétation, est observée dans les jachères, elle est caractérisée par un processus d'installation des composantes herbeuses et d'enrichissement en éléments ligneux. C'est le développement d'une végétation herbeuse vers une formation naturelle. Ceci dépend de paramètres favorisants qui pourraient être naturels, sans aucune intervention de l'homme, demandant des actions comme la mise en défends du secteur, l'embroussaillement, le reboisement et autres. Ainsi, la densité moyenne (nombre d'individus par hectare) de la zone d'étude est de 260 individus par hectare dont Guiera senegalensis représente plus de $70 \%$, alors qu'elle est de
326,11 individus/ha dans la jachère et 194 individus/ha dans le champ soit la moitié. En effet Guiera senegalensis c'est une espèce pionnière signalée dans plusieurs travaux effectuée dans notre zone d'étude. La régénération de cette espèce, selon Ichaou (2000) cité par (Rabiou, 2014) est assurée principalement par la multiplication végétative. Ce type de régénération, comportant plusieurs formes telles le marcottage, le drageonnage et le rejet de souche (Bellefontaine, 2005), se réalise à partir de tiges couchées ou de souches d'arbres abattus. Le recouvrement moyen de la zone d'étude est de $6,08 \%$. Mais la jachère présente un recouvrement moyen supérieur $(9,11 \%)$ au recouvrement de la zone d'étude. La hauteur moyenne de la végétation ligneuse est de 1,28 $\pm 0,64 \mathrm{~m}$. La hauteur moyenne la plus élevée est observée dans la jachère 1,39 $\pm 0,63$ alors qu'elle est de 1,01 $\pm 0,5$ dans le champ. Ce résulte montre que l'abandon de champ enclenche un processus dynamique de succession post-cultural des communautés végétales dans les jachères protégées de Banizoumbou. Le potentiel ligneux de Banizoumbou présente les distributions des formations végétales unimodales caractéristiques des peuplements à structure inéquienne. La structure en diamètre présente une distribution asymétrique négative, caractéristique des peuplements mono-spécifique avec prédominance d'individus jeunes ou de petits diamètres, avec un paramètre de forme $c$ de la distribution théorique de Weibull égale à 1,79. En effet, cette structure semble être une structure d'un peuplement en pleine régénération. Mais en réalité c'est une structure forcée car les individus de gros diamètre sont systématiquement coupés comme bois de chauffe commercialisé par la population locale. De cette façon il subsiste toujours des individus jeunes appelés tiges d'avenir. Selon Ouédraogo (2006), Dan Guimbo et al. (2010), et Rabiou et al. (2014), la prédominance des individus de petit diamètre est une structure souvent observée pour les écosystèmes non perturbés. Les individus de petit diamètre assurent l'avenir des formations naturelles, tandis que ceux de gros diamètre résultant de la sélection naturelle sont des semenciers qui assurent l'avenir de peuplement à travers la production des graines (Morou, 2010). En examinant de plus près les structures en diamètre de peuplement de jachère et du champ on se rend compte que le peuplement le plus stable est celui de jachère avec un paramètre de forme $c$ de la distribution de Weibull égal à 0,79 . Par contre, dans les champs le paramètre de forme $c$ est égale à 1,71 . Après l'abandon de champ le peuplement ligneux n'est plus donc soumis au défrichement ce qui induit un processus dynamique et progressif de peuplement 
ligneux. Par contre les champs sont exposés chaque année à un défrichement et émondage de son

\section{CONCLUSION}

Un schéma synthétique général des successions postculturales des jachères protégées de Banizoumbou a été proposé dans le présent travail. II présente le remplacement des types biologiques les uns par les autres au cours de la reconstitution du milieu. Les variations observées par rapport à ce schéma dans les divers écosystèmes (jachère et champ) sont interprétées en fonction de l'âge de champ et des jachères. En zone de jachères protégées de Banizoumbou tout au moins, la richesse des ligneux augmente tout au long de la succession, tandis que celle des herbacés diminue très rapidement après la phase d'augmentation initiale. Cela s'explique bien dans le cadre de la théorie dite de la «perturbation intermédiaire» qui stipule que les plus fortes

\section{RÉFÉRENCES BIBLIOGRAPHIQUE}

Achard F., Hiernaux P., Banoin M., 2001. Les jachères fourragères naturelles et améliorées en Afrique de l'Ouest, La jachère en Afrique tropicale - Ch. Floret, R. Pontanier John Libbey Eurotext, Paris @ 2001, pp. 201-239.

Barbier N., Couteron P., Lejoly J., Deblauwe V. et Lejeune O., 2006. Self-organised vegetation patterning as fingerprint of climate and human impact on semiarid ecosystems. Journal of Ecology 94 : 537-547.

Bellefontaine R., 2005. Pour de nombreux ligneux, la reproduction sexuée n'est pas la seule voie : analyse de 875 cas - Texte introductif ; $5 p$. Sécheresse 1(3).

Bogaert J. et Mahamane A., 2005. Écologie du paysage : cibler la configuration et l'échelle spatiale. Annales des Sciences Agronomiques du Bénin, 7: 39-68.

Braun-Blanquet J., 1932. Plant sociology. The study of plant communities. New Works ; Londres ; McGray Hill ; 439p.

Camara Y., 1997. Effet du raccourcissement du temps de jachère sur la régénération de $P$. erinaceus en Haute Casamance (Sénégal). Mémoire d'ingénieur des Eaux et Forêts, école nationale des cadres ruraux, République du Sénégal, 32 p. + annexes.

Cissé M., Hiernaux P., et Diarra L., 1993. Intégration agro-pastorale au Sahel: dynamique et potentiel fourrager des jachères, in Floret \&Serpantié (éd., 1993) : pp. 405-413. peuplement ligneux.

diversités correspondent à des taux de ressource modérément limitant, car ces taux peuvent survenir plus ou moins rapidement en fonction des organismes considérés et des caractéristiques des milieux considérés. Les écosystèmes sous régime de jachère courte qui se mettent en place dans le contexte actuel sont en revanche régis par d'autres contraintes qui correspondent aux perturbations récurrentes d'un défrichement répété et du cortège de pressions anthropiques qui l'accompagnent. Ces modifications entraînent une banalisation des flores, sans perte de productivité dans un premier temps, et une redistribution de l'importance des types de potentiels floristiques dans la reconstitution de milieu.

Couteron, P., Lejeune, O., 2001. Periodic spotted patterns in semi-arid vegetation explained by a propagation-inhibition model. Journal of Ecology, Vol. 89 (4), p. 616- 628.

Dan Guimbo I., Mahamane A. et Ambouta K. J.-M., 2010, Peuplement des parcs à Neocarya macrophylla (Sabine) Prance et à Vitellaria paradoxa (Gaertn. C.F.) dans le sud-ouest nigérien : diversité, structure et régénération. International journal of biological and chemical science, 4(5) : 1706-1720.

Delabre É., 1998. Caractérisation et évolution d'écosystèmes anthropisés sahéliens: les milieuxpost-culturaux du sud-ouest nigérien, th., écologie, univers. Pierre-et-Marie-Curie, Paris-VI, $289 \mathrm{p}$.

Fournier A., Hien M. et Millogo-Rasolodimby J., 2001. Les jachères de moins de cinq ans en savane soudanienne : richesse Joristique, structure de la végétation. Cas de Bondoukuy (Ouest du Burkina Faso), in Floret \&Pontanier (éd., 2000) : vol. 1,pp. 390-399.

Hien M., 1996. La reconstitution postculturale de la végétation en savane soudanienne dans la région de Bondoukuy (Burkina Faso). Les jachères de moins de six ans: flore, persistance des adventices, lien avec le milieu et son utilisation, Mémoire DEA, université de Ouagadougou, $95 \mathrm{p}$.

Hiernaux P., 1983. Recherche d'une solution aux problèmes de l'élevage dans le delta intérieur 
du Niger au Mali, vol. 1, Les pâturages de la zone d'étude, Addis Abeba-Sevare, CipeaOdem, 133p.

Mahamane A., 2005. Études floristique, phytosociologique et phytogéographique de la végétation du Parc Régional du W du Niger. Thèse de Doctorat ès Sciences Agronomiques et Ingénierie Biologique. Université Libre de Bruxelles, $536 \mathrm{p}$.

Mariama D., 2009. Impacts de la variabilité climatique sur la dynamique de la strate herbacée dans la commune de Dantiandou. Mémoire de DEA, université de Niamey, 74p.

Morou B., 2010. Impacts de l'occupation des sols sur I'habitat de la girafe au Niger et enjeux pour la sauvegarde du dernier troupeau de girafes de l'Afrique de l'Ouest. Thèse de Doctorat, Université Abdou Moumouni, $184 \mathrm{p}$.

Ouédraogo A., 2006. Diversité et dynamique de végétation ligneuse de la partie orientale $\mathrm{du}$ Burkina Faso. Thèse de doctorat, Université de Ouagadougou Burkina Faso, 195p + Annexe.

Rabiou H., Inoussa M. M., Bakasso Y., Diouf A., Moussa M. B., Mahamane A., Saidou I. S., Saadou M., Lykke A. M., 2014. Structure de la population de Boscia senegalensis (Pers) Lam. Ex Poir. suivant la toposéquense dans la commune de Simiri (Niger). Journal of Animal \&Plant Sciences, 23 (3) : 3657-3669.
Randrianjafy Z. J. N., 2009. Dynamique de végétation, destinées des "tanety" et forêts brûlées 8p.

Raunkiaer C., 1934. The life forms of plants and statistical plant geography. Clarendon, Oxford.

Rondeux J., 1999. La mesure des arbres et des peuplements forestiers. Gembloux, Belgique, Les Presses agronomiques de Gembloux, 2e édition, $521 \mathrm{p}$.

Serpantié G., Thomas J.N. et Douanio M., 2000. «Évolution de la place de la jachère en savane soudanienne cotonnière. Le cas de la région de Bondoukui », in Floret \& Pontanier (éd., 2000) : vol. 1, pp. 80-91.

Song H., Yu Q., Shang K. K., Yang T. \& Da L. J., 2011. The spatio-temporal pattern of historical disturbances of an evergreen broadleaved forest in East China : a dendrological analysis. Plant Ecology, 212: 1313-1325.

Tijani A. D., 2008. Érosion éolienne dans le Damagaram Est (Sud-Est du Niger) : Paramétrisation, quantification et moyens de lutte: thèse de doctorat : $171 \mathrm{p}$.

White F., 1986. La végétation de l'Afrique, Paris, OrstornUnesco, $384 \mathrm{p}$.

Yossi H., 1996. Dynamique de la végétation postculturale en zone soudanienne au Mali, th. doct., population environnement, Isfra, université de Bamako, $154 \mathrm{p}$. 